\title{
On some fundamental theorems of contingent equations in connection with the control problems
}

By

\author{
Norio KIKUCHI*
}

\section{Introduction}

While the ordinary differential equations corresponding to vector fields, the contingent equations correspond to set-valued fields approximately known up to a given accuracy.

Hence in this case we have to deal with the more general theory of differential inequalities.

On the other hand this problems arise in the control theory. That is, assume that there are given the following relations,

$$
\begin{aligned}
d x(t) / d t & =f(t, x(t), u(t)), \\
u(t) & \in Q(t, x(t)),
\end{aligned}
$$

where $f$ is a mapping of $\left[t_{0}, t_{0}+a\right] \times R^{n} \times R^{r}$ into $R^{n}$ and $Q(t, x)$ is a compact set in $R^{r}$ for every $(t, x) \in\left[t_{0}, t_{0}+a\right] \times R^{n}$ and $u(t) \in R^{r}$ is a measurable function on $\left[t_{0}, t_{0}+a\right]$. A solution $x(t)$ of this problem also satisfies the following relation,

$$
d x(t) / d t \in f(t, x(t), Q(t, x(t))) .
$$

By using a suitable implicit function theorem, the converse problem can be considered. Hence, first we consider the contingent equations and investigate some fundamental theorems similar to those of the differential equations and finally we apply these theorems to the control problems.

Received July 20, 1967.

Communicated by M. Hukuhara.

* Department of Mathematics, Kôbe University. 
Our approach in this paper is mainly due to that of T. Ważewski's papers [5], [6].

The author wishes here to express his thanks to Professor Masuo Hukuhara for his warm encouragement. This paper is deeply indebted to his papers [1], [2] for the set-valued function theorems.

\section{Notations and definitions}

The notations used in this paper are the followings.

Let $X$ be a metric space. The distance between two points $x, y \in X$ is denoted by $\operatorname{dist}(x, y)$. The distance between a point $x \in X$ and a set $A \subset X$ is defined by

$$
\operatorname{dist}(x, A)=\inf \{\operatorname{dist}(x, y) ; y \in A\} .
$$

The distance between $A$ and $B \subset X$ is defined by

$$
\operatorname{dist}(A, B)=\inf \{\operatorname{dist}(x, y) ; x \in A, y \in B\} \text {. }
$$

For $\delta>0$ and a set $A \subset X$ we put

$$
\begin{aligned}
& U(A, \delta)=\{x \in X ; \operatorname{dist}(x, A)<\delta\}, \\
& \bar{U}(A, \delta)=\{x \in X ; \operatorname{dist}(x, A) \leqq \delta\},
\end{aligned}
$$

and

$$
U(A,-\delta)=\{x \in X ; U(x, \delta) \subset A\}
$$

By

$$
\operatorname{Comp}(X), \quad(\operatorname{resp} \cdot \operatorname{Conv}(X))
$$

we denote the collection of all nonempty compact (resp. compact and convex) subsets of $X$. For two $A, B \in \operatorname{Comp}(X)$, the Hausdorff distance between $A$ and $B$ is denoted by $\operatorname{Dist}(A, B)$, where

$$
\operatorname{Dist}(A, B)=\inf \{\delta>0 ; U(A, \delta) \supset B, U(B, \delta) \supset A\} \text {. }
$$

This Hausdorff distance makes $\operatorname{Comp}(X)$ into a metric space.

For a set $A$ in $X$ we denote by bdry $A$ the boundary of $A$.

We denote by $R^{m}$ an $m$-dimensional Euclidean space with the usual scalar product $x \cdot y$ for every $x$ and $y$ and the induced norm $|x|$, $\left(|x|^{2}=x \cdot x,|x| \geqq 0\right)$ and by $I$ the compact interval $\left[t_{0}, t_{0}+a\right]$ in $R^{1}$. 
For a set $A$ in $R^{m}$ we put

$$
|A|=\operatorname{Dist}(O, A) \text {, }
$$

where $O$ is the origin of $R^{m}$.

A map $F(t, x)$ defined on $I \times R^{m}$ into $\operatorname{Comp}\left(R^{m}\right)$ will be called an orientor field. If $F(t, x)$ reduces to a single point for every $(t, x)$, then we have to do with a vector field as a special case of an orientor field.

Definition 1. A function $F(t) \in \operatorname{Comp}(X)$ defined on a topological space $T$ is said to be upper (resp. lower) semi-continuous at $t_{0}\left(t_{0} \in T\right)$ if for every $\varepsilon>0$ we can find some neighborhood of $t_{0}$, say $V$, such that

$$
U\left(F\left(t_{0}\right), \varepsilon\right) \supset F(t) \quad\left(\operatorname{resp} . U(F(t), \varepsilon) \supset F\left(t_{0}\right)\right)
$$

for all $t \in V$. When $F(t)$ is upper (resp. lower) semi-continuous at every point of $T, F(t)$ is said to be upper (resp. lower) semi-continuous on $T$. A function $F(t)$ is said to be continuos at $t_{0}$ (resp. on $T$ ) when $F(t)$ is upper and lower semi-continuous at $t_{0}$ (resp. on $T$ ).

Definition 2. If a function $F(t) \in \operatorname{Comp}(X)$ defined on a measurable space $E$ is such that, for every $C \in \operatorname{Comp}(X)$, the set $\{t \in E$; $F(t) \subset C\}$ is measurable, then $F$ is said to be measurable on $E$.

Definition 3. For a sequence of subsets (in $X)\left\{A_{k}\right\}(k=1,2, \cdots)$ we define

$$
\begin{aligned}
& \lim _{k \rightarrow \infty} \inf A_{k}=\left\{x \in X ; \lim _{k \rightarrow \infty} \operatorname{dist}\left(x, A_{k}\right)=0\right\}, \\
& \lim _{k \rightarrow \infty} \sup A_{k}=\left\{x \in X ; \lim _{k \rightarrow \infty} \operatorname{dist}\left(x, A_{k}\right)=0\right\},
\end{aligned}
$$

and

$$
\lim _{k \rightarrow \infty} A_{k}=\lim _{k \rightarrow \infty} \inf A_{k}=\lim _{k \rightarrow \infty} \sup A_{k},
$$

when $\quad \lim _{k \rightarrow \infty} \inf A_{k}=\lim _{k \rightarrow \infty} \sup A_{k}$.

It is known [1] that these sets

$$
\lim _{k \rightarrow \infty} \inf A_{k}, \quad \lim _{k \rightarrow \infty} \sup A_{k}
$$

are closed. 
Let $F(t) \in \operatorname{Conv}\left(R^{m}\right)$ be defined and measurable on a measurable set $E$. We denote by $|F|(t)$ a scalar function $|F(t)|$. If $|F|(t)$ is integrable on $E$, then the Lebesgue integral $\int_{E} F(t) d t$ has been defined and some properties similar to those of a vector valued Lebesgue integral have been investigated in [2]. In this case we say that $F$ is integrable.

$\operatorname{By}$ mesble $(E)$ we denote the sets of all functions measurable on $E$. The abbreviation a.e.t $\in E$ means almost every $t$ in $E$.

We consider the function $N(t, x)$ defined on $I \times R^{n}$ and in $\operatorname{Comp}\left(R^{n}\right)$ for each fixed $(t, x)$ such that $N(t, x)$ carries every bounded set in $I \times R^{n}$ into a bounded set in $R^{n}$.

On the function $N(t, x)$ which satisfies the above conditions, the following additional conditions will be imposed as needed.

Hypothesis $\mathbb{H}(\mathbb{N}) \cdot N(t, x)$ is measurble in $t$ for each fixed $x \in R^{n}$ and upper semi-continuous in $x$ for each fixed $t \in I$.

Hypothesis $\mathbb{H}_{1}(\mathbb{N}), N(t, x)$ is measurable in $t$ for each fixed $x \in R^{n}$ and continuous in $x$ for each fixed $t \in I$.

Hypothesis $\mathbb{H}_{2}(\mathbb{N}), N(t, x)$ is upper semi-continuous in $(t, x)$.

Hypothesis $\mathbb{H}_{3}(\mathbb{N}) 。 N(t, x)$ is continuous in $(t, x)$.

\section{Propositions}

In [3], [4] we have proved the following propositions which will be used in the following.

Proposition 1 . Let $F(t) \in \operatorname{Comp}\left(R^{n}\right)$ be defined and measurable on a measurable set $E$. Suppose that meas $(E)<\infty$. Then there exists a measurable function $f(t) \in R^{n}$ on $E$ such that

$$
f(t) \in F(t)
$$

for every $t \in E$.

Proposition 2. Let $F(t, u) \in \operatorname{Comp}\left(R^{n}\right)$ be defined on $I \times R^{r}$ and measurable in $t$ for each fixed $u \in R^{r}$ and continuous in $u$ for each fixed $t \in I$. 
Let $Q(t) \in \operatorname{Comp}\left(R^{r}\right)$ be bounded and measurable on $I$. Let $R(t)$ denote the set $F(t, Q(t))$ and $y(t) \in R^{n}$ be a measuralbe function such that

$$
y(t) \in R(t) \quad \text { for every } t \in I .
$$

Then there exists a measurable function $u(t)$ such that $u(t) \in Q(t)$ and $y(t) \in F(t, u(t))$ for every $t \in I$.

Proposition 3. Let $\left\{F_{k}(t)\right\} \quad(k=1,2, \cdots)$ be a sequence of compact and convex set (in $R^{n}$ ) valued functions defined and integrable on $E$, and suppose that there is an integrable function $F_{0}(t) \in \operatorname{Conv}\left(R^{n}\right)$ such that

$$
F_{k}(t) \subset F_{0}(t) \quad(k=1,2, \cdots) \text { on } E,
$$

then $\lim _{k \rightarrow \infty} \sup F_{k}(t)$ is integrable on $E$ and the relation

$$
\lim _{k \rightarrow \infty} \sup \int_{E} F_{k}(t) d t \subset \int_{E} \lim _{k \rightarrow \infty} \sup F_{k}(t) d t
$$

holds.

Proposition 4. Let $F(t, x) \in \operatorname{Comp}\left(R^{n}\right)$ be defined on $I \times R^{n}$ and measurable in $t$ for each fixed $x \in R^{n}$ and upper semi-continuous in $x$ for each fixed $t \in I$. Then $F(t, x(t))$ is measurable in $t$ for each measurable function $x(t) \in R^{n}$.

We cite the fundamental Theorem which was proved in [1] by defining the topological degree of a compact and convex set valued function.

Fundamental Theorem. Let $F(t) \in \operatorname{Conv}\left(R^{n}\right)$ be defined on a closed set $E$ of $R^{m}$. Then there exists a monotone decreasing sequence of $\left\{F_{k}(t)\right\}(k=1,2, \cdots)$ such that each $F_{k}(t) \in \operatorname{Conv}\left(R^{n}\right)$ is continuous on $E$ and $F_{k}(t)$ converges to $F(t)$ and $F(t)$ is contained in the interior of $F_{k}(t)$ for each $t \in E$ and $k$.

Remark. If $F(t)$ is bounded on $E$, then $F_{k}(t)$ can be assumed to be bounded on $E$. 


\section{Contingent equations}

Let $x(t) \in R^{n}$ be a function defined on an interval $J \subset R^{1}$. Let $t_{0} \in J$ and put

$$
p(t)=\frac{x(t)-x\left(t_{0}\right)}{t-t_{0}}
$$

A set $M \subset R^{n}$ is said a contingent derivative of $x(t)$ at $t_{0}$ and is denoted by $D^{*} x\left(t_{0}\right)$, when, for $t \rightarrow t_{0}$,

$$
\lim \sup |p(t)|<+\infty
$$

and when $M$ consists of $c \in R^{n}$ for which there exists a sequence $\left\{t_{k}\right\}$ such that

$$
t_{k} \in J, \quad t_{k} \neq t_{0}, \quad t_{k} \rightarrow t_{0}, \quad p\left(t_{k}\right) \rightarrow c .
$$

By abs. $\operatorname{cont}(J)$ and $\operatorname{cont}(J)$ we denote the sets of all functions absolutely continuous and continuous on each compact set contained in the interval $J$, respectively.

We introduce two (equivalent) definitions of a trajectory of an orientor field $F(t, x)$.

Definition 4. (of Marchaud). A function $x=x(t)$ defined on an interval $J$ will be called a trajectory of $F(t, x)$ if

$$
\begin{aligned}
& x(t) \in \operatorname{cont}(J), \quad \\
& D^{*} x(t) \subset F(t, x(t)) \text { for almost every } t \in J .
\end{aligned}
$$

Definition 5. (of Ważewski). A function $x=x(t)$ defined on an interval $J$ will be called a trajectory of $F(t, x)$ if

$$
\begin{aligned}
& x(t) \in \text { abs. } \operatorname{cont}(J), \\
& d x(t) / d t \in F(t, x(t)) \quad \text { for almost every } t \in J .
\end{aligned}
$$

Theorem 0. Suppose that $F(t, x) \in \operatorname{Conv}\left(R^{n}\right)$ satisfies $\mathrm{H}(\mathrm{F})$. Then Definition 4 and 5 are equivalent.

Proof. Assume that $x(t)$ satisfies the relation of Definition 4 . Let $L$ be a compact interval of $J$. As $x(t)$ is continuous, $x(t)$ is bounded on $L$ and there exists $C \in \operatorname{Comp}\left(R^{n}\right)$ such that $D^{*} x(t) \subset$ $F(t, x(t)) \subset C$ for almost every $t \in L$. Hence we conclude that $x(t)$ 
satisfies a Lipschitz condition and therefore $x(t)$ is absolutely continuous on $L$. A derivative $d x(t) / d t$ exists almost everywhere in $J$. Consequently the relation

$$
d x(t) / d t \in D^{*} x(t) \subset F(t, x(t))
$$

holds for almost every $t$ in $J$.

Next assume that $x(t)$ satisfies the relation of Definition 5 . As $F(t, x(t))$ is measurable on $J$ by Proposition 4 , the relation

$$
x(t)-x(\tau) \in \int_{\tau}^{t} F(t, x(t)) d t
$$

holds for every every $\tau, t \in J$.

Therefore

$$
D^{*} x(\tau) \subset F(\tau, x(\tau))
$$

holds for almost every $\tau, t \in J$.

Definition 6. Let $A \in \operatorname{Comp}\left(R^{n}\right)$ and $\tau, T \in\left[t_{0}, t_{0}+a\right]$.

By $T(A, \tau, F)$ we denote the family of trajectories of $F$ such that $x(\tau) \in A$, by $Z(A, \tau, F)$ the union of the graphs of functions belonging to $T(A, \tau, F)$.

By $S(A, \tau, F, T)$ we denote the set $Z(A, \tau, F) \cap\{t=T\}$. When $\tau$ is equal to $t_{0}$, we write briefly $T(A, F), Z(A, F)$ and $S(A, F, T)$ for $T(A, \tau, F), Z(A, \tau, F)$ and $S(A, \tau, F, T)$ respectively.

\section{Some fundamental theorems of contingent equations}

In this chapter we consider only the function $F(t, x)$ defined on $I \times R^{n}$ and in $\operatorname{Conv}\left(R^{n}\right)$ for each fixed $(t, x)$ such that for every $y \in F(t, x)$ there holds

$$
x \cdot y \leqq C\left(|x|^{2}+1\right) \quad(C>0),
$$

and $F(t, x)$ carries every bounded set in $I \times R^{n}$ into a bounded set in $R^{n}$.

$\mathbf{1}^{\circ}$. In this section we consider the case when $F$ satisfies Hypothesis $\mathrm{H}(\mathrm{F})$. 
In [4] we have proved the following theorems.

Theorem 1. Suppose that $F$ satisfies Hypothesis $\mathrm{H}(\mathrm{F})$. Then for every $x_{0} \in R^{n}$ there exists a trajectory (on $I$ ) in $T\left(x_{0}, F\right)$.

Theorem 2. Suppose that $F$ satisfies Hypothesis $\mathrm{H}(\mathrm{F})$. Then for every $A \in \operatorname{Comp}\left(R^{n}\right), T(A, F)$ is compact in the topology of the uniformly convergence.

Further we can prove the following theorems.

Theorem 3. Let $A$ be in $\operatorname{Comp}\left(R^{n}\right)$. Then the section $S(A, F, \tau)$ is in $\operatorname{Comp}\left(R^{n}\right)$ for every $\tau \in I$.

Proof. All trajectories $x(t)$ of $F$ through $A$ are contained in a bounded set.

Indeed, if $z(t)=|x(t)|^{2}+1$, then

$$
d z(t) / d t \leqq 2 C z(t)
$$

hence

$$
z(t) \leqq\left(|A|^{2}+1\right) \exp (2 C a)
$$

i.e.

$$
|x(t)|^{2} \leqq\left(|A|^{2}+1\right) \exp (2 C a)-1
$$

Hence the section $S(A, F, \tau)$ is a bounded set for each $\tau \in I$.

In order to show that each section is closed we assume that $\left\{x_{k}\right\} \subset S(A, F, \tau)(k=1,2, \cdots)$, where $x_{k} \rightarrow x_{0}$. Since $x_{k} \in S(A, F, \tau)$, there is some trajectory $x_{k}(t)$ of $F$ through $x_{k}\left(t_{0}\right) \in A$ and $x_{k}(\tau)=x_{k}$.

By Theorem 2 we can assume that $\left\{x_{k}(t)\right\}$ converges uniformly on $I$ to a trajectory $x(t)$ of $F$. Since $A$ is closed, $x_{k}\left(t_{0}\right) \rightarrow x\left(t_{0}\right) \in A$, so $x_{0}=x(\tau) \in S(A, F, \tau)$. Hence $S(A, F, \tau)$ is compact in $R^{n}$.

For each $A \in \operatorname{Comp}\left(R^{n}\right)$ we consider the following mapping of $I$ into $\operatorname{Comp}\left(R^{n}\right)$;

$$
S_{A} ; t \rightarrow S(A, F, t)
$$

Theorem 4. $S_{A}$ is continuous on $I$.

Proof. Let $\tau \in I$, then $S(A, F, \tau) \in \operatorname{Comp}\left(R^{n}\right)$. Also $Z(A, F)$ is 
in $\operatorname{Comp}\left(I \times R^{n}\right)$, and then $F(t, x)$ is bounded on $Z(A, F)$. Let $M=\sup |F(t, x)|$ on $Z(A, F)$.

For every $\varepsilon$

$$
S(A, F, \tau+s) \subset U(S(A, F, \tau), \varepsilon)
$$

holds for $s,|s|<\alpha=\varepsilon M^{-1}$.

Hence $S_{A}$ is upper semi-continuous on $I$.

Next we prove that $S_{A}$ is lower semi-continuous on $I$.

For every $\varepsilon>0$

$$
S(A, F, \tau+s+u) \subset U(S(A, F, \tau+s), \varepsilon)
$$

holds for all $s$ and $u,|u|<\alpha$.

If we put $u=-s$, we have

$$
S(A, F, \tau) \subset U(S(A, F, \tau+s), \varepsilon)
$$

for $s,|s|<\alpha$, which implies that $S_{A}$ is lower semi-continuous on $I$.

Hence the continuity of $S_{A}$ has been proved.

For each $t \in I$ we consider the following mapping of $\operatorname{Comp}\left(R^{n}\right)$ into $\operatorname{Comp}\left(R^{n}\right)$;

$$
S_{i} ; A \rightarrow S(A, F, t) .
$$

Theorem 5. The mapping $S_{t}$ is upper semi-continuous on $\operatorname{Comp}\left(R^{n}\right)$ uniformly with respect to $t$.

Proof. Let $A \in \operatorname{Comp}\left(R^{n}\right)$ and $\varepsilon$ be any positive number. We want to find $\delta>0$ such that

$$
S_{t}(B) \subset U\left(S_{t}(A), \varepsilon\right)
$$

for every compact set $B$, Dist $(B, A)<\delta$ and for every $t$ on $I$.

$\bar{U}(A, 1)$ is a compact set and hence $Z(\bar{U}(A, 1), F)$ is also a compact set. Hence there exists a constant $M>0$ such that

$$
|F(t, x)| \leqq M \quad \text { on } Z(\bar{U}(A, 1), F) .
$$

Consequently for every $\varepsilon>0$ we can find a positive number $\eta$ (sufficiently small)

$$
\eta<\min (1, \varepsilon / 2, \varepsilon / 2 M)
$$


such that for each $\tau \in I$

$$
S_{\tau+t}(B) \subset U\left(S_{\tau}(A), \varepsilon\right)
$$

holds for every $B, \operatorname{Dist}(A, B)<\eta$ and every $t$ on $[\tau, \tau+\eta] \cap I$.

This $\eta$ can be taken independently of $\tau$.

Next we shall prove that there exists $\delta>0$ such that

$$
S_{t}(B) \subset U\left(S_{t}(A), \varepsilon\right)
$$

holds for every $B \in \operatorname{Comp}\left(R^{n}\right), \operatorname{Dist}(A, B)<\delta$ and for every $t \in\left[t_{0}, t_{0}+\delta\right]$.

Indeed, if this were not true, then for every $k(k=1,2, \cdots)$ there is a $B_{k} \in \operatorname{Comp}\left(R^{n}\right)$ and $t_{k} \in\left[t_{0}, t_{0}+1 / k\right]$ such that

$$
\begin{aligned}
& S_{t_{k}}\left(B_{k}\right) \mp U\left(S_{t_{k}}(A), \varepsilon\right), \\
& \operatorname{Dist}\left(B_{k}, A\right)<1 / k .
\end{aligned}
$$

In other words, there exists a sequence $\left\{b_{k}\right\}, b_{k} \in B_{k}$ and a sequence $\left\{t_{k}\right\}$ on $I$ such that

$$
\operatorname{dist}\left(b_{k}, A\right)<1 / k \text {, }
$$

and for some solution $x_{k}(t)$ through $b_{k}$ we have

$$
\operatorname{dist}\left(x_{k}\left(t_{k}\right), S_{t_{k}}(A)\right) \geqq \varepsilon .
$$

Since $b_{k} \in \bar{U}(A, 1)$, we can select a subsequence of $\left\{x_{k}(t)\right\}$ which converges uniformly to a trajectory $x(t)$ of $F$. Without changing the notation we let $\left\{x_{k}(t)\right\}$ converge to $x(t)$ uniformly.

Furthermore, since $I$ is a compact set, $\left\{t_{k}\right\}$ can also be assumed to converge to some $\bar{t} \in I$. Since $x\left(t_{0}\right) \in A$ can be verified, $x(t) \in S_{t}(A)$ holds for every $t$ on $I$.

By Theorem 3

$$
\operatorname{Dist}\left(S_{\bar{t}}(A), S_{t_{k}}(A)\right)<\varepsilon / 2
$$

holds for sufficiently large $k$, and hence

$$
\operatorname{dist}\left(x_{k}\left(t_{k}\right), S_{\bar{t}}(A)\right) \geqq \varepsilon / 2 \text {. }
$$

Since $\left\{x_{k}(t)\right\}$ is a normal family, $\left\{x_{k}\left(t_{k}\right)\right\}$ converges to $x(\bar{t})$. 
Consequently $\operatorname{dist}\left(x(\bar{t}), S_{\bar{t}}(A)\right) \geqq \varepsilon / 2$ holds, which contradicts the fact that

$$
x(t) \in S_{t}(A)
$$

holds for every $t$ on $I$.

Next we define the set

$D=\left\{\tau \in\left(t_{0}, t_{0}+a\right]\right.$; for every $\varepsilon>0$ there exists $\delta>0$ such that $S_{t}(B) \subset U\left(S_{t}(A), \varepsilon\right)$ holds for every $B, \operatorname{Dist}(B, A)<\delta$ and every $t$ on $\left.\left[t_{0}, \tau\right]\right\}$.

As stated above this set is not empty.

Let $T=\sup D\left(T>t_{0}\right)$, and we shall show that

$$
T=t_{0}+a \in D \text {. }
$$

If $T<t_{0}+a$, there exist $a^{\prime}\left(0<a^{\prime}<\eta\right)$ and $T^{\prime}$ such that

$$
t_{0} \leqq T^{\prime}<T<T^{\prime}+a^{\prime} \leqq t_{0}+a,
$$

and

$$
S\left(C, T^{\prime}, F, t\right) \subset U\left(S_{t}(A), \varepsilon\right)
$$

holds for every $C \in \operatorname{Comp}\left(R^{n}\right), \operatorname{Dist}\left(C, S_{T^{\prime}}(A)\right)<\eta$ and for every $t$ on $\left[T^{\prime}, T^{\prime}+a^{\prime}\right] \cap I$.

Since $T^{\prime}<T$, we can find $\delta>0$ such that

$$
\operatorname{Dist}\left(S_{t}(B), S_{t}(A)\right)<\eta \text {, }
$$

holds for every $B \in \operatorname{Comp}\left(R^{n}\right), \operatorname{Dist}(B, A)<\delta$ and for every $t \in\left[t_{0}, T^{\prime}\right]$.

Since we can take $S_{T^{\prime}}(B)$ for $C$,

$$
S_{t}(B)=S\left(S_{T^{\prime}}(B), T^{\prime}, F, t\right) \subset U\left(S_{t}(A), \varepsilon\right)
$$

holds for every $t$ on $\left[t_{0}, T^{\prime}+a^{\prime}\right] \cap I$, which contradicts the definition of $T$.

$t_{0}+a \in D$ can be similarly verified.

Let $K(t) \in \operatorname{Comp}\left(R^{n}\right)$ be defined and upper semi-continuous on $I$.

We say that $K(t)$ is attainable from $A$ along trajectories of $F$ if there exists $x=x(t) \in T(A, F)$ such that $x\left(t_{0}\right) \in A$ and $x(\tau) \in K(\tau)$ for some $\tau \in I$. 
Theorem 6. Suppose that $K(t)$ satisfies the conditions stated above. Also suppose that $K(t)$ is attainable from $A$ along trajectories of $F$. Then there exists a trajectory $\bar{x}(t)$ along which $K(t)$ is attainable from $A$ in the minimum time $\bar{t}$. Furthermore, if $K(t)$ is continuous on $I$, the point $(\bar{t}, \bar{x}(\bar{t}))$ is on the boundary of $Z(A, F)$.

Proof. Consider the set of $\tau \in I$ such that

$$
x(\tau) \in K(\tau)
$$

for some $x(t) \in T(A, F)$. This set is not empty by hypothesis. Since $I$ is compact, we can select a sequence $\left\{t_{k}\right\}(k=1,2, \cdots)$ from this set such that $\left\{t_{k}\right\}$ converges to $\bar{t}$-the greatest lower bound for this set. Let $x_{k}(t) \in T(A, F)$ be a trajectory corresponding to $t_{k}$.

From the compactness of $T(A, F)$ we can assume that $\left\{x_{k}(t)\right\}$ converges uniformly to $\bar{x}(t) \in T(A, F)$.

Making use of the equi-continuity of $T(A, F)$ and the upper semi-continuity of $K(t)$, we conclude that

$$
\bar{x}\left(t_{0}\right) \in A \text { and } \bar{x}(\bar{t}) \in K(\bar{t}) .
$$

In the case when $K(t)$ is continuous, this point $(\bar{t}, \bar{x}(\bar{t}))$ is on $\operatorname{bdry} Z(A, F)$. Indeed, if $(\bar{t}, \bar{x}(\bar{t}))$ is an interior point of $Z(A, F)$, there exists $\delta>0$ such that

$$
U=\{(t, x) ; \bar{t}-\delta \leqq t<\bar{t},|x-\bar{x}(\bar{t})| \leqq \delta\}
$$

is contained in $Z(A, F)$. By the continuity of $K(t)$ we conclude that $\{(t, K(t)) ; t \in I\}$ contains a point of $U$.

Hence there exists $t_{1} \in I\left(t_{1}<\bar{t}\right)$ such that

$$
x_{1}\left(t_{1}\right) \in K\left(t_{1}\right)
$$

for some $x_{1}(t) \in T(A, F)$, which contradicts the definition of $\bar{t}$.

$2^{\circ}$. In this section we consider the case when $F$ satisfies Hypothesis $\mathrm{H}_{1}(\mathrm{~F})$.

Theorem 7. Let $A \in \operatorname{Comp}\left(R^{n}\right)$ be a connected set. Then the section $S(A, F, \tau) \in \operatorname{Comp}\left(R^{n}\right)$ is a connected set for each $\tau \in I$. 
Proof. Suppose that $S(A, F, \tau)$ is not connected for some $\tau \in I$, and then $S(A, F, \tau)$ can be expressed as the union of two disjoint compact sets, i.e.,

$$
S(A, F, \tau)=S_{1} \cup S_{2},
$$

where $S_{1}, S_{2}$ are compact sets and $S_{1} \cap S_{2}=\phi$.

Let $A_{i}(i=1,2)$ be the set

$$
\left\{q \in A: S_{i} \cap S(q, F, \tau) \neq \phi\right\} .
$$

The sets $A_{1}, A_{2}$ can be verified to be compact by the compactness of $T(A, F)$. Since $A$ is connected and $A=A_{1} \cup A_{2}$, we can find a point $p$ such that $p \in A_{1} \cap A_{2}$.

Let $S_{i}^{\prime}=S_{i} \cap S(p, F, \tau)(i=1,2)$, and $S_{1}^{\prime}$ and $S_{2}^{\prime}$ are disjoint compact sets. Hence $\operatorname{dist}\left(S_{1}^{\prime}, S_{2}^{\prime}\right)=d>0$.

Let $H$ be the compact set

$$
H=\left\{x ; \operatorname{dist}\left(x, S_{1}^{\prime}\right)=(1 / 2) d\right\}
$$

in the plane $t=\tau$. By definition of $H$

$$
H \cap S_{\imath}^{\prime}=\phi(i=1,2)
$$

hold.

Let $x=\varphi_{i}(t)(i=1,2)$ be a trajectory which passes through a point $p=\left(t_{0}, x_{0}\right), x_{0} \in A$ and a point $q_{i}$ of $S_{i}^{\prime}$, respectively.

Let $D$ be a subdivision of $I$ :

$$
t_{0}<t_{1}<t_{2}<\cdots<t_{k}=t_{0}+a^{\prime}=\tau .
$$

Take $\bar{t} \in I, \bar{x}=\varphi_{1}(\bar{t})$, and then for some $i_{0} t_{i_{0}} \leqq \bar{t}<t_{i_{0}+1}$ holds. We put $\bar{x}_{i_{0}}=\bar{x}$. The set

$$
\left.C_{i_{0}}(t)=\left\{u ; \operatorname{dist}\left(\varphi_{1}^{\prime}(t), F\left(t, \bar{x}_{i_{0}}\right)\right)\right)=\operatorname{dist}\left(\varphi_{1}^{\prime}(t), u\right)\right\}
$$

can be verified to be in $\operatorname{Comp}\left(R^{n}\right)$ and measurable on $I$. Hence the lexicographic maximum $u_{i_{0}}(t)$ of $C_{i_{0}}(t)$ is measurable and satisfies the relation

$$
u_{i_{0}}(t) \in C_{i_{0}}(t)
$$

for all $t, \bar{t}_{i_{0}} \leqq t<t_{i_{0}+1}$.

For $\bar{t} \leqq t \leqq t_{i_{0}+1}$ we define 


$$
\bar{x}+\int_{\bar{t}}^{t} u_{i_{0}}(t) d t=x(t, \bar{t}, \bar{x})
$$

and put

$$
\bar{x}_{i_{0}+1}=x\left(t_{i_{0}+1}, \bar{t}, \bar{x}\right) \text {. }
$$

We define inductively $\left\{\bar{x}_{i}\right\}$ and $\left\{u_{i}(t)\right\}\left(i=i_{0}, i_{0}+1, \cdots, k\right)$ as follows. Suppose that we have defined $\bar{x}_{i}$ and then for $t \leqq t \leqq t_{i+1}$ we define

$$
\bar{x}(t, \bar{t}, \bar{x})=\bar{x}_{i}+\int_{t_{i}}^{t} u_{i}(t) d t
$$

where $u_{i}(t)$ is a measurable function which is the lexicographic maximum of the set

$$
C_{i}(t)=\left\{u ; \operatorname{dist}\left(\varphi_{1}^{\prime}(t), F\left(t, \bar{x}_{i}\right)\right)=\operatorname{dist}\left(\varphi_{1}^{\prime}(t), u\right)\right\} .
$$

This function $x(t, \bar{t}, \bar{x})$ can be verified to be continuous in $(\bar{t}, \bar{x})$ by the continuity of $F(t, x)$ in $x$. When $\bar{t} \in I, \bar{x}=\varphi_{2}(\bar{t})$, we similarly define $x(t, \bar{t}, \bar{x})$.

Here we define

$$
\psi(s)= \begin{cases}\varphi_{1}\left(t_{0}+a^{\prime}|s|\right) & (-1 \leqq s \leqq 0) \\ \varphi_{2}\left(t_{0}+a^{\prime}|s|\right) & (0<s \leqq 1) .\end{cases}
$$

Let $\left\{D_{k}\right\}(k=1,2, \cdots)$ be a sequence of subdivisions of $I\left(D_{k}\right.$; $\left.t_{0}<t_{1}^{(k)}<t_{2}^{(k)}<\cdots<t_{l_{k}}^{(k)}=t_{0}+a^{\prime}\right)$ such that a norm $\delta\left(D_{k}\right)=\max _{0 \leqq i<l_{k}}\left(t_{i+1}^{(k)}-t_{i}^{(k)}\right)$ tends to zero as $k \rightarrow \infty$.

Let $x_{k}(t, \bar{t}, \bar{x})$ be the corresponding $x(t, \bar{t}, \bar{x})$ to $D_{k}$. A curve

$$
B_{k}(s)=\left(\tau, x_{k}\left(\tau, t_{0}+a^{\prime}|s|, \psi(s)\right)\right)
$$

is a continuous curve which connects two points $q_{1}$ and $q_{2}$. Hence $B_{k}(s)$ must pass through $H$ at some point $q_{k}=\left(\tau, z_{k}\right)$.

Consequently we can find $-1 \leqq s_{k} \leqq 1$ (say, $t_{i_{0}-1}^{(k)} \leqq t_{0}+a^{\prime}\left|s_{k}\right|<t_{i_{0}}^{(k)}$ ) such that

$$
z_{k}=x_{k}\left(\tau, t_{0}+a^{\prime}\left|s_{k}\right|, \psi_{r}\left(s_{k}\right)\right) .
$$

For $t_{0} \leqq t \leqq t_{0}+a^{\prime}\left|s_{k}\right|$ we define

$$
\chi_{k}(t)= \begin{cases}\varphi_{1}(t) & \left(s_{k} \leqq 0\right) \\ \varphi_{2}(t) & \left(s_{k}>0\right)\end{cases}
$$


and for $t_{0}+a^{\prime}\left|s_{k}\right| \leqq t \leqq t_{0}+a^{\prime}$

$$
\chi_{k}(t)=x_{k}\left(t, t_{0}+a^{\prime}\left|s_{k}\right|, \psi\left(s_{k}\right)\right) .
$$

The function $\chi_{k}(t)$ thus defined is continuous on $\left[t_{0}, t_{0}+a^{\prime}\right]$ and the relations

and

$$
\chi_{k}\left(t_{0}\right)=x_{0}
$$

$$
d x_{k}(t) / d t \in F\left(t, y_{k}(t)\right)
$$

hold, where

$$
y_{k}(t)= \begin{cases}\varphi_{1}(t) & \left(t_{0} \leqq t \leqq t_{0}+a^{\prime}\left|s_{k}\right|, s_{k} \leqq 0\right) \\ \varphi_{2}(t) & \left(t_{0} \leqq t \leqq t_{0}+a^{\prime}\left|s_{k}\right|, s_{k}>0\right) \\ x_{k}\left(t_{0}+a^{\prime}\left|s_{k}\right|, t_{0}+a^{\prime}\left|s_{k}\right|, \psi\left(s_{k}\right)\right) \quad\left(t_{0}+a^{\prime}\left|s_{k}\right|<t<t_{i_{0}}^{(k)}\right) \\ x_{k}\left(t_{i}^{(k)}, t_{0}+a^{\prime}\left|s_{k}\right|, \psi_{r}\left(s_{k}\right)\right) \quad\left(t_{i}^{(k)} \leqq t<t_{i+1}^{(k)}, i_{0} \leqq i \leqq l_{k}-2\right) \\ x_{k}\left(t_{l_{k}-1}^{(k)}, t_{0}+a^{\prime}\left|s_{k}\right|, \psi\left(s_{k}\right)\right) & \left(t_{l_{k}-1}^{(k)} \leqq t \leqq t_{l_{k}}\right) .\end{cases}
$$

$\left\{\chi_{k}(t)\right\}$ can be verified to be a normal family.

From the construction of $\left\{y_{k}(t)\right\}$ we can assume that $\left\{x_{k}(t)\right\}$ with. $\left\{y_{k}(t)\right\}$ converges uniformly to a continuous function $x(t)$.

Since

$$
\begin{aligned}
\chi_{k}(t)-x_{0} & \in \int_{t_{0}}^{t} F\left(t, y_{k}(t)\right) d t, \\
x(t)-x_{0} & \in \lim _{k \rightarrow \infty} \sup \int_{t_{0}}^{t} F\left(t, y_{k}(t)\right) d t \\
& \subset \int_{t_{0}}^{t} \lim _{k \rightarrow \infty} \sup F\left(t, y_{k}(t)\right) d t \\
& \subset \int_{t_{0}}^{t} F(t, x(t)) d t
\end{aligned}
$$

holds.

On the other hand this function $x(t)$ is absolutely continuous since all the $\chi_{k}(t)$ satisfy the same Lipschitz condition. Hence $x\left(t_{0}\right)=x_{0}$ and

$$
d x(t / d t \in F(t, x(t))
$$

hold for almost every $t \in\left[i_{0}, \tau\right]$.

Since $\chi_{k}(\tau) \in H$ and $H$ is a closed set, we conclude $x(\tau) \in H$, which contradicts the fact that $H \cap S_{\imath}=\phi(i=1,2)$.

Theorem 8. Every $Q\left(t_{1}, x_{1}\right)$ on bdry $Z(A, F)$ can be peripherally 
attainable from $\operatorname{bdry} A$, i.e., there exists a trajectory $\varphi(t) \in T(A, F)$ such that $(t, \varphi(t)) \in \operatorname{bdry} Z(A, F)$ for every $t \in\left[t_{0}, t_{1}\right]$.

Proof. Take $t_{2}$ such that $t_{0}<t_{2}<t_{1}$. We first prove that there exists a trajectory $\varphi(t)$ which connects the point $\left(t_{2}, \varphi\left(t_{2}\right)\right) \in \operatorname{bdry} Z(A, F)$ and $Q\left(t_{1}, x_{1}\right)$. If $Q^{\prime}\left(t_{2}, \psi\left(t_{2}\right)\right)$ doesn't belong to bdry $Z(A, F)$ for some trajectory $\psi(t) \in T(A, F), Q^{\prime}$ is an interior point of $Z(A, F)$. We take an exterior point $Q_{1}$ sufficiently near $Q$. Let $\overline{Q Q}_{1}$ be the segment which connects $Q$ and $Q_{1}$. The cross section $S\left(\overline{Q Q}_{1}, t_{1}, F, t_{2}\right)$ of $Z\left(\overline{Q Q_{1}}, t_{1}, F\right)$ by the hyperplane $t=t_{2}$ is a continuum by Theorem 7 . Therefore there is a trajectory which connects of $S\left(A, F, t_{2}\right)$ and $\overline{Q Q}_{1}$. Since $Q$ is a boundary point of $Z(A, F)$, for every positive integer $k$ we can find an exterior point $S_{k}\left(t_{1}, y_{k}\right)$ such that $y_{k} \in U\left(x_{1}, 1 / k\right)$. As stated above, we can find a trajectory $\varphi_{k}(t) \in T(A, F)$ such that $\left(t_{2}, \varphi_{k}\left(t_{2}\right)\right)$ belongs to bdry $Z(A, F)$ and $\left(t_{1}, \varphi_{k}\left(t_{1}\right)\right)$ belongs to $\overline{Q S}_{k}$. By Theorem 2 we can assume that $\left\{\varphi_{k}(t)\right\}$ converges uniformly to $\varphi(t) \in T(A, F)$. Therefore this $\varphi(t) \in T(A, F)$ connects $\left(t_{2}, \varphi\left(t_{2}\right)\right) \in$ bdry $Z(A, F)$ and $Q$.

Let $D$ be a subdivision of an interval $\left(t_{0}, t_{1}\right)$ :

$$
t=\alpha_{0}<\alpha_{1}<\alpha_{2}<\cdots<\alpha_{N}=t_{1} .
$$

As stated above, there is a trajectory $\varphi(t)$ starting from $Q$ such that $\left(\alpha_{N-1}, \varphi\left(\alpha_{N-1}\right)\right)$ belongs to $\operatorname{bdry} Z(A, F)$ and similarly there is a trajectory $\varphi(t)$ starting from $\left(\alpha_{N-1}, \varphi\left(\alpha_{N-1}\right)\right)$ such that $\left(\alpha_{N-2}, \varphi\left(\alpha_{N-2}\right)\right)$ belongs to $\operatorname{bdry} Z(A, F)$.

In this way we can find a trajectory $\varphi(t)$ such that $\left(\alpha_{k}, \varphi\left(\alpha_{k}\right)\right)$ $(k=1,2, \cdots, N-1)$ all belong to $\operatorname{bdry} Z(A, F)$.

Take a sequence of subdivisions $\left\{D_{k}\right\}(k=1,2, \cdots)$ such that every division point of $D_{k}$ is that of $D_{k+1}$ for all $k$ and the maximum length of subinterval of $D_{k}$ tends to zero as $k \rightarrow \infty$.

Corresponding to $D_{k}$ we can find a trajectory $\varphi_{k}(t)$ which has the property stated above.

By Theorem 2 we can assume that $\left\{\varphi_{k}(t)\right\}$ converges uniformly to a trajectory $\varphi(t) \in T(A, F)$ which connects $A$ and $Q$. 
This $(t, \varphi(t))$ belongs to $\operatorname{bdry} Z(A, F)$ for every $t \in\left[t_{0}, t_{1}\right]$ from the construction.

$3^{\circ}$. In this section we consider the case when $F$ satisfies Hypothesis $\mathrm{H}_{2}(\mathrm{~F})$.

Let $F(t, x) \in \operatorname{Conv}\left(R^{n}\right)$ satisfy $\mathrm{H}_{2}(\mathrm{~F})$. Here we denote

$$
\left(|A|^{2}+1\right) \exp (2 C a)-1
$$

by $H^{2}(H>0)$. By taking a sufficiently large $C$, we can assume that $H>1$. We define

$$
\bar{F}(t, x)= \begin{cases}F(t, x), & x \leqq H \\ F(t, H x /|x|), & x>H .\end{cases}
$$

$\bar{F}(t, x)$ defined as above can be verified to be upper semi-continuous in $(t, x)$ and bounded on $I \times R^{n}$.

Hence by the fundamental Theorem cited in Chapter 2 we can find a sequence $\left\{F_{k}(t, x)\right\}(k=1,2, \cdots)$ such that each $F_{k}(t, x) \in \operatorname{Conv}\left(R^{n}\right)$ satisfies $\mathrm{H}_{3}\left(\mathrm{~F}_{k}\right)$ and contains $\bar{F}(t, x)$ in its interior and $\left\{F_{k}(t, x)\right\}$ converges to $\bar{F}(t, x)$ monotone decreasingly.

Theorem 9. Let $\left\{A_{k}\right\}(k=1,2, \cdots)$ be a sequence of $\operatorname{Comp}\left(R^{n}\right)$ such that $\left\{A_{k}\right\}$ converges to $A \in \operatorname{Comp}\left(R^{n}\right)$ monotone decreasingly. Then the following relations

$$
\begin{aligned}
& \bigcap_{k=1}^{\infty} T\left(A_{k}, F_{k}\right)=T(A, F), \\
& \bigcap_{k=1}^{\infty} Z\left(A_{k}, F_{k}\right)=Z(A, F),
\end{aligned}
$$

and

hold.

$$
\bigcap_{k=1}^{\infty} S\left(A_{k}, F_{k}, \tau\right)=S(A, F, \tau) \quad \text { for every } \tau \in I
$$

Proof. As $F_{k}(t, x)(k=1,2, \cdots)$ contains $\bar{F}(t, x)$ for every point $(t, x) \in I \times R^{n}$ and $A_{k}$ contains $A$, then $T\left(A_{k}, F_{k}\right)$ contains $T(A, \bar{F})$ for each $k$, and hence the relation

$$
\bigcap_{k=1}^{\infty} T\left(A_{k}, F_{k}\right) \supset T(A, \bar{F})
$$

holds. 
Next we shall prove that every $x(t)$ in $\bigcap_{k=1}^{\infty} T\left(A_{k}, F_{k}\right)$ belongs to $T(A, \bar{F})$. Since $x(t)$ belongs to $\bigcap_{k=1}^{\infty} T\left(A_{k}, F_{k}\right)$, the following relations

$$
x(t)-x\left(t_{0}\right) \in \int_{t_{0}}^{t} F_{k}(t, x(t)) d t \text { for each } k
$$

and

$$
x\left(t_{0}\right) \in A
$$

hold.

By Proposition 3 we conclude

$$
\begin{aligned}
x(t)-x\left(t_{0}\right) & \in \lim _{k \rightarrow \infty} \sup \int_{t_{0}}^{t} F_{k}(t, x(t)) d t \\
& \subset \int_{t_{0}}^{t} \lim _{k \rightarrow \infty} \sup F_{k}(t, x(t)) d t \\
& =\int_{t_{0}}^{t} \bar{F}(t, x(t)) d t .
\end{aligned}
$$

Therefore

$$
d x(t) / d t \in \bar{F}(t, x(t)) \text { a.e. } t \in I,
$$

and

$$
x\left(t_{0}\right) \in A \text {. }
$$

Hence $x(t) \in T(A, F)$ holds, which proves

$$
\bigcap_{k=1}^{\infty} T\left(A_{k}, F_{k}\right) \subset T(A, \bar{F}) .
$$

For $\bar{F}(t, x)$ the same relation as $F(t, x)$, i.e., $x \cdot y \leqq C\left(|x|^{2}+1\right)$, holds for every $y \in \bar{F}(t, x)$. From this condition we can conclude that all solutions of

$$
d \bar{x} / d t \in \bar{F}(t, x) \text { and } x\left(t_{0}\right) \in A
$$

satisfy $|x(t)| \leqq H$ on $I$. Indeed, if $z(t)=|x(t)|^{2}+1$, then $d z(t) / d t \leqq$ $2 C z(t)$, hence $z(t) \leqq\left(|A|^{2}+1\right) \exp (2 C a)$, i.e. $|x(t)| \leqq H$. In $|x| \leqq H$, $\bar{F}(t, x)$ and $F(t, x)$ coincide. Hence a solution $x(t)$ for

$$
d x / d t \in \bar{F}(t, x) \text { and } x\left(t_{0}\right) \in A
$$

is also that for

$$
d x / d t \in F(t, x) \text { and } x\left(t_{0}\right) \in A .
$$


Consequently $T(A, \bar{F})$ and $T(A, F)$ coincide.

Hence we have proved that the relation

$$
\bigcap_{k=1}^{\infty} T\left(A_{k}, F_{k}\right)=T(A, F)
$$

holds.

We can similarly prove the relations

$$
\bigcap_{k=1}^{\infty} Z\left(A_{k}, F_{k}\right)=Z(A, F),
$$

and

$$
\bigcap_{k=1}^{\infty} S\left(A_{k}, F_{k}, \tau\right)=S(A, F, \tau) .
$$

Theorem 10. Let $A \in \operatorname{Comp}\left(R^{n}\right)$ be a connected set. Then the section $S(A, F, \tau) \in \operatorname{Comp}\left(R^{n}\right)$ is a connected set for every $\tau \in I$.

Proof. Let $\left\{F_{k}(t, x)\right\}(k=1,2, \cdots)$ be a sequence as stated above. Then by Theorem 7 each $S\left(A, F_{k}, \tau\right)$ is a continuum for every $\tau \in I$. On the other hand, by Theorem $9 S(A, F, \tau)=\bigcap_{k=1}^{\infty} S\left(A, F_{k}, \tau\right)$, which shows that $S(A, F, \tau)$ is a continuum.

Theorem 11. Every $P$ on bdry $Z(A, F)$ can be peripherally attainable from bdry $A$.

Proof. Let $\left\{F_{k}(t, x)\right\}(k=1,2, \cdots)$ be a sequence as stated above. Since $Z\left(A, F_{k}\right)$ converges to $Z(A, F)$ monotone decreasingly, $\operatorname{bdry} Z\left(A, F_{k}\right)$ is known to converge to $\operatorname{bdry} Z(A, F)$, and hence we can take a sequence of points $\left\{P_{k}\right\}(k=1,2, \cdots), P_{k} \in \operatorname{bdry} Z\left(A, F_{k}\right)$, which converges to $P$.

By Theorem 8 there exists a sequence of trajectories $\left\{x_{k}(t)\right\}$ such that $\left(t, x_{k}(t)\right)$ runs through only $\operatorname{bdry} Z\left(A, F_{k}\right)$ and connnects bdry $A$ and $P_{k} \in \operatorname{bdry} Z\left(A, F_{k}\right)$. Since $x_{k}(t)$ can be verified to be a normal family, we can assume that $\left\{x_{k}(t)\right\}$ converges to a function $x(t)$ uniformly. Since $x_{k}(t)$ is a trajectory of $F_{k}(t, x)$, the following relation

$$
x_{k}(t)-x_{k}\left(t_{0}\right) \in \int_{t_{0}}^{t} F_{k}\left(t, x_{k}(t)\right) d t
$$

holds. 
By the upper semi-continuity of $F(t, x)$ in $x$ and Proposition 3 we conclude that

$$
\begin{aligned}
x(t)-x\left(t_{0}\right) & \in \lim _{k \rightarrow \infty} \sup \int_{t_{0}}^{t} F_{k}\left(t, x_{k}(t)\right) d t \\
& \subset \int_{t_{0}}^{t} \lim _{k \rightarrow \infty} \sup F_{k}\left(t, x_{k}(t)\right) d t \\
& \subset \int_{t_{0}}^{t} F(t, x(t)) d t,
\end{aligned}
$$

and since $x(t)$ is absolutely continuous,

$$
d x(t) / d t \in F(t, x(t)) \quad \text { a.e. } t \in I
$$

and

$$
x\left(t_{0}\right) \in \operatorname{bdry} A .
$$

Hence we can find a trajectory $x(t)$ which connects bdry $A$ and $P$. Also $Z\left(A, F_{k}\right) \supset Z(A, F)$ for each $k$ and $\left(t, x_{k}(t)\right) \in \operatorname{bdry} Z\left(A, F_{k}\right)$ holds for every $t \in I$.

Hence $x(t)$ does not belong to the interior of $Z(A, F)$.

On the other hand, since $x(t)$ belongs to $T(A, F),(t, x(t)) \in$ bdry $Z(A, F)$ holds for every $t \in I$.

Remark. Theorem 11 can also be proved similarly to Theorem 8 by using Theorem 10 .

$4^{\circ}$. In this section we consider the case when $F$ satisfies Hypothesis $\mathrm{H}_{3}(\mathrm{~F})$.

Theorem 12. Suppose that $x(t) \in T(A, F)$ is a trajectory which lies on bdry $Z(A, F)$ for every $t$ on $I$. Then $d x(t) / d t$ is on bdry $F(t, x(t))$ for almost every $t$ on $I$.

Proof. Let $d x(\bar{t}) / d t$ belong to the interior of $F(\bar{t}, x(\bar{t}))$ for some $\bar{t}$ on $I$ and so belong to $U(F(\bar{t}, x(\bar{t})),-3 \varepsilon)$ for some $\varepsilon>0$.

By the continuity of $F(t, x)$ in $(t, x)$ there exists $\delta>0$ such that

$$
F(t, x) \supset U(F(\bar{t}, x(\bar{t})),-\varepsilon)
$$

for every $t$ and $x,|t-\bar{t}| \leqq \delta,|x-x(\bar{t})| \leqq \delta$.

Hence for every $\tau$ sufficiently near $\bar{t}(\tau<\bar{t})$, the set 


$$
\begin{aligned}
& \{(t, x(\tau)+m(t-\tau)) ; m \in U(F(\bar{t}, x(\bar{t})),-\varepsilon), t \in I\} \\
& \cap\{(t, x) ;|t-\bar{t}| \leqq \delta,|x-x(\bar{t})| \leqq \delta\}
\end{aligned}
$$

is contained in $Z(A, F)$.

On the other hand, from the differentiability of $x(t)$ at $\bar{t}$

$$
\left(x(\bar{t})-x\left(t_{2}\right)\right) /\left(\bar{t}-t_{2}\right) \in U(F(\bar{t}, x(\bar{t})),-2 \varepsilon)
$$

holds for some $t_{2}$ sufficiently near $\bar{t}, t_{2}<\bar{t}$.

Consequently

$$
x(\bar{t}) \in x\left(t_{2}\right)+U(F(t, x(t)),-2 \varepsilon)\left(\bar{t}-t_{2}\right),
$$

and hence $x(\bar{t})$ belongs to the interior of $Z(A, F)$, which contradicts that $x(\bar{t})$ is on bdry $Z(A, F)$.

Theorem 13. Suppose that $F(t, x)$ has an interior point at every point $(t, x) \in I \times R^{n}$. Then there exists a continuously differentiable trajectory $x(t)$ of $F$, i.e., for $x(t)$

$$
d x(t) / d t \in F(t, x(t))
$$

holds for every $t \in I$ and

$$
x\left(t_{0}\right) \in A \text {. }
$$

Proof. Let $f(t, x)$ be a bary-center of $F(t, x)$. This function $f(t, x)$ is continuous on $I \times R^{n}$.

Hence there is a continuously differentiable function $x(t)$ such that

$$
d x(t) / d t=f(t, x(t)) \text { holds for every } t \text { on } I
$$

and

$$
x\left(t_{0}\right) \in A .
$$

This function satisfies the wanted relations;

$$
d x(t) / d t=f(t, x(t)) \in F(t, x(t)) \text { for every } t \text { on } I
$$

and

$$
x\left(t_{0}\right) \in A
$$


Remark. The method of taking out a bary-center of $F(t, x)$ has been used in [1] by defining the topological degree of a compact and convex set valued function.

\section{Control systems of contingent equations}

Introduce the variable $u=\left(u_{1}, u_{2}, \cdots, u_{r}\right) \in R^{r}$.

Let

$$
F(t, x, u)
$$

be a mapping of $I \times R^{n} \times R^{r}$ into $\operatorname{Comp}\left(R^{n}\right)$ and

$$
Q(t, x)
$$

be a mapping of $I \times R^{n}$ into $\operatorname{Comp}\left(R^{r}\right)$.

The variable $u$ is called the control.

Definition 7. By a control system $S(F, Q)$ we mean a pair: a field $F(t, x, u)$ and a field $Q(t, x), Q(t, x)$ is called the control domain of $S(F, Q)$.

Definition 8. A function $x=x(t)$ defined on an interval $I$ is said to be a trajectory of $S(F, Q)$ if $x(t) \in$ abs. $\operatorname{cont}(I)$ and if there rexists a control function $u(t)$ such that

$$
\begin{aligned}
d x(t) / d t & \in F(t, x(t), u(t)) \quad \text { a.e. } t \in I, \\
u(t) & \in \operatorname{mesble}(I), \\
u(t) & \in Q(t, x(t)) .
\end{aligned}
$$

We shall make the following assumptions.

1) $F(t, x, u)$ is measurable in $t$ for each fixed $(x, u) \in R^{n} \times R^{r}$ and continuous in $(x, u)$ for each fixed $t \in I$ and $F(t, x, u)$ carries every bounded set in $I \times R^{n} \times R^{r}$ into a bounded set in $R^{n}$.

2) $Q(t, x)$ is measurable in $t$ for each fixed $x \in R^{n}$ and upper semicontinuous in $x$ for each fixed $t \in I$, and $Q(t, x)$ carries every bounded set in $I \times R^{n}$ into a bounded set in $R^{r}$.

3) $R(t, x)=F(t, x, Q(t, x))$ is in $\operatorname{Conv}\left(R^{n}\right)$ for each $(t, x) \in I \times R^{n}$ and for every $(t, x) \in I \times R^{n}, \quad x \cdot y \leq C\left(|x|^{2}+1\right) \quad(C>0)$ holds for every $y \in R(t, x)$. 
The set $R(t, x)$ will be called the control counterdomain of $S(F, Q)$. We have an orientor field $R(t, x)$ defined on $I \times R^{n}$, which we will call the orientor field associated to the control system $S(F, Q)$.

By passing from a control system $S(F, Q)$ (depending on the control $u$ ) to the associated orientor field $R(t, x)$ (independent of $u$ ), a trajectory of $S(F, Q)$ can be considered to be a trajectory of $R(t, x)$.

The inverse problem consists of the following.

Suppose that we know a trajectory $x=x(t)$ of an orientor field $R(t, x)$ associated with a control system $S(F, Q)$. We would like to find the corresponding control function $u(t)$. To do this we have to find a measurable function $u(t)$ satisfying conditions:

$$
\begin{aligned}
d x(t) / d t & \in F(t, x(t), u(t)) \quad \text { a.e. } t \in I, \\
u(t) & \in Q(t, x(t)) .
\end{aligned}
$$

Proposition 2 answers to this problem.

We shall consider the control problem for the contingent equation.

Let $A \in \operatorname{Comp}\left(R^{n}\right)$, and $K(t) \in \operatorname{Comp}\left(R^{n}\right)$ be defined and upper semi-continuous on $I . C(t, x)$ is a real function defined on $I \times T(A, F)$ and is continuous in $(t, x)$. We say that a control $u(t)$, defined for $t_{0} \leqq t \leqq \bar{t}, \bar{t} \in I$, transfers $A$ to $K(t)$ if one of the trajectories $x(t)$ corresponding to $u(t)$ satisfies the relations $x\left(t_{0}\right) \in A$ and $x(\bar{t}) \in K(\bar{t})$. We restrict ourselves to the problem of finding a control function $u(t)$ which transfers $A$ to $K(t)$ and which minimizes the cost functional

$$
C(t, x) \text {, }
$$

where $x$ is one of the solution corresponding to $u(t)$ and $t$ represents a value of $t$ such that $x(t) \in K(t)$.

Theorem 14. Suppose that the conditions stated above are satisfied. Also suppose that there exists at least one control $u(t)$ which transfers $A$ to $K(t)$ on $I$. Then there exists an optimal control, i.e., a measurable function $u^{*}(t)$ for which one of the corresponding solutions, $x^{*}(t)$, with initial condition $x^{*}\left(t_{0}\right) \in A$ attains $K\left(t^{*}\right)$ for some $t^{*}$ in $I$ and 


$$
\inf C(t, x)=C\left(t^{*}, x^{*}\right),
$$

where, in addition, $u^{*}(t) \in Q\left(t, x^{*}(t)\right)$.

Proof. Now consider the set of all the $x(t)$ satisfying

$$
\begin{gathered}
d x(t) / d t \in F(t, x(t), u(t)) \quad \text { a.e. } t \in I, \\
x\left(t_{0}\right) \in A \text { and } x(\bar{t}) \in K(\bar{t})
\end{gathered}
$$

for some $\bar{t} \in I$, where, in addition, $u(t) \in Q(t, x(t))$ for some control $u(t)$.

Since one such solution exists by hypothesis, this set is not empty. Consequently we can select a sequerce of trajectories $\left\{x_{k}(t)\right\}(k=1$, $2, \cdots$ ) on $I$, with

$$
C\left(t_{k}, x_{k}\right)
$$

decreasing monotonically to inf $C(t, x)$, where $t_{k}$ represents a value of $t$ such that $x_{k}(t) \in K(t) . \quad x_{k}(t)$ satisfies the following relations

$$
d x_{k}(t) / d t \in R\left(t, x_{k}(t)\right) \text { a.e. } t \in I,
$$

and $x_{k}\left(t_{0}\right) \in A$. By the compactness of $T(A, R)$ we conclude that

$$
\begin{aligned}
d x^{*}(t) / d t & \in R\left(t, x^{*}(t)\right) \text { a.e. } t \in I, \\
x^{*}\left(t_{0}\right) & \in A,
\end{aligned}
$$

where $x^{*}(t)$ is a limit function of a subsequence of $\left\{x_{k}(t)\right\}$. Also since $I$ is a compact interval, we can select a further subsequence (without changing the notation) such that $\left\{t_{k}\right\}$ converges to some $t^{*}$ in $I$. Further making use of the equi-continuity of $\left\{x_{k}(t)\right\}$ and the upper semi-continuity of $K(t)$, we conclude that $x^{*}\left(t_{0}\right) \in A$ and $x^{*}\left(t^{*}\right) \in K\left(t^{*}\right)$.

Also by the continuity of $C(t, x),\left\{C\left(t_{k}, x_{k}\right)\right\}$ approaches $C\left(t^{*}, x^{*}\right)$ as $k \rightarrow \infty$, and hence inf $C(t, x)=C\left(t^{*}, x^{*}\right)$.

By Proposition 2 we can select a measurable function $u^{*}(t)$ such that

$$
d x^{*}(t) / d t \in F\left(t, x^{*}(t), u^{*}(t)\right) \text { a.e. } t \in I,
$$

and $u^{*}(t) \in Q\left(t, x^{*}(t)\right)$ on $I$.

Hence $u^{*}(t)$ is an optimal control. 
Theorem 15. Suppose that the conditions stated in Theorem 14 are satisfied and further suppose that $K(t)$. is continuous on $I$. We restrict ourselves to a time optimal problem, i.e., to the case of $C(t, x)=t$. Then there exists an optimal trajectory $x^{*}(t)$ along which $K(t)$ is peripherally attainable.

Proof. By Theorem 6 the point $\left(t^{*}, x^{*}\left(t^{*}\right)\right)$ in the proof of Theorem 14 is on bdry $Z(A, R)$.

By Theorem $8\left(t, x^{*}(t)\right)$ can be assumed to be on bdry $Z(A, R)$ for every $t \in\left[t_{0}, t^{*}\right]$.

The family of trajectories of $S(F, Q)$ is denoted by $\{F, Q\}$. We define the bang-bang kernel $Q_{1}(t, x)$ of control domain $Q(t, x)$ by the formula

$$
Q_{1}(t, x)=\{u ; u \in Q(t, x), F(t, x, u) \subset \operatorname{bdry} R(t, x)\} .
$$

Theorem 16. In addition to the conditions in Theorem 14, we assume that $R(t, x)$ is continuous in $(t, x)$. Then there exists an optimal trajectory $x(t)$ which belongs to the family $\left\{F, Q_{1}\right\}$.

Proof. This theorem follows from the definition of $\left\{F, Q_{1}\right\}$ and Theorem 12.

\section{REFERENCES}

[1] Hukuhara, M., Sur l'application semi-continue dont la valeur est un compact convexe, RIMS-11, Res. Inst. Math. Sci., Kyoto Univ., 1966.

[2] - Intégration des applications mesurables dont la valeur est un compact convexe, RIMS-15, Res. Inst. Math. Sci., Kyoto Univ., 1966.

[3] Kikuchi, N., Existence of optimal controls, RIMS-20, Res. Inst. Math. Sci., Kyoto Univ., 1967.

[4] Control problems of contingent equation, Publ. RIMS, Kyoto Univ. Ser. A, 3 (1967), 85-99.

[5] Ważewski, T., Sur une condition équivalente à l'équation au contingent, Bull. Acad. Polon. Sci., Sér. Sci. Math. Astr. Phys. 9 (1961), 865-867.

[6] - On an optimal control problem, Differential Equations and Their Applications: Proceedings of the Conference held in Prague, September 1962 (1963), 229-242. 
